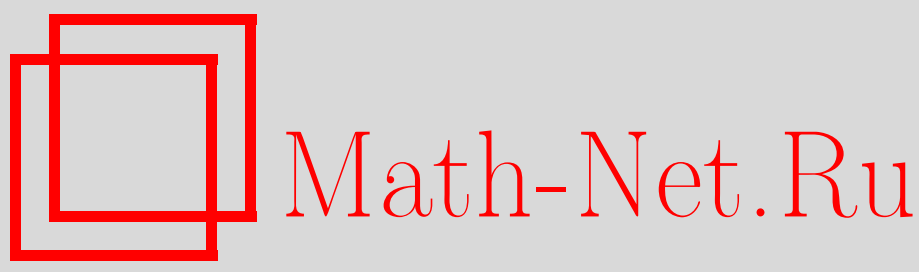

В. Л. Хацкевич, О законе больших чисел для нелинейных средних случайных величин, Изв. вузов. Матем., 2019, номер 8, 79-87

DOI: https://doi.org/10.26907/0021-3446-2019-8-79-87

Использование Общероссийского математического портала Math-Net.Ru подразумевает, что вы прочитали и согласны с пользовательским соглашением

http://www . mathnet.ru/rus/agreement

Параметры загрузки:

IP: 3.93 .64 .190

26 апреля 2023 г., 03:06:44

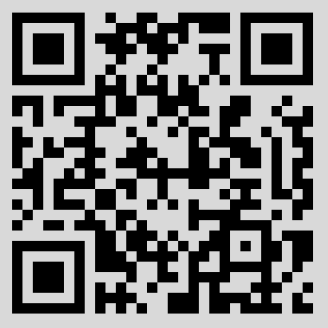


Известия вузов. Математика

2019, № 8, c. 79-87 https://kpfu.ru/science/nauchnye-izdaniya/ivrm

e-mail: izvuz.matem@kpfu.ru

\title{
В.Л. ХАЦКЕВИЧ
}

\section{О ЗАКОНЕ БОЛЬШИХ ЧИСЕЛ ДЛЯ НЕЛИНЕЙНЫХ СРЕДНИХ СЛУЧАЙНЫХ ВЕЛИЧИН}

\begin{abstract}
Аннотация. Рассмотрены варианты закона больших чисел относительно средних степенных, средних геометрических и средних гармонических систем случайных величин. Установлены различные виды сходимости указанных средних к предельным значениям.
\end{abstract}

Ключевые слова: случайная величина, нелинейная средняя, закон больших чисел.

УДК: 519.244

DOI: 10.26907/0021-3446-2019-8-79-87

\section{ВВЕДЕНИЕ}

Приведем общую постановку рассматриваемой проблемы из ([1], гл. 6, §31). Пусть дана последовательность случайных величин $\xi_{1}, \xi_{2}, \ldots, \xi_{n}, \ldots$ Рассматриваются случайные величины $\eta_{n}$, являющиеся функциями от $n$ первых случайных величин последовательности $\eta_{n}=$ $f_{n}\left(\xi_{1}, \xi_{2}, \ldots, \xi_{n}\right)$. Если существует такая последовательность постоянных $a_{1}, a_{2}, \ldots, a_{n}, \ldots$, что при любом $\varepsilon>0$

$$
\lim _{n \rightarrow \infty} P\left\{\left|\eta_{n}-a_{n}\right|<\varepsilon\right\}=1,
$$

то говорят, что данная последовательность $\xi_{1}, \xi_{2}, \ldots, \xi_{n}, \ldots$ подчиняется закону больших чисел (коротко ЗБЧ) с заданными функциями $f_{n}$. Также говорят, что последовательность $f_{n}$ асимптотически постоянна, или стабилизируется к числовой последовательности $a_{n}$. Традиционно ограничиваются случаем, когда $f_{n}-$ средние арифметические величин $\xi_{1}, \xi_{2}, \ldots$, $\xi_{n}, \ldots$, а числовую последовательность $a_{n}$ составляют математические ожидания либо медианы $f_{n}$. О некоторых направлениях развития ЗБЧ для нелинейных функций $f_{n}$ будет сказано в заключении данной работы при сравнении результатов.

Классический ЗБЧ, развитый Чебышевым, Марковым, Ляпуновым и их последователями, характеризует предельное поведение средних арифметических случайных величин, когда $f_{n}=\frac{1}{n} \sum_{i=1}^{n} \xi_{i}$. Однако в статистике и других прикладных науках используются не только средние арифметические, но и другие средние - средние геометрические, средние степенные, средние гармонические (например, [2]). В данной работе показывается, что для таких средних систем случайных величин имеют место аналоги ЗБЧ. Акцент делается на доказательстве сходимости в пространствах суммируемых функций.

Поступила в редакцию 27.06.2018, после доработки 27.06.2018. Принята к публикации 19.12.2018 
Как известно, математическим ожиданием случайной величины $\xi$, обладающей функцией распределения $F_{\xi}(x)$, называется значение интеграла $E \xi=\int_{-\infty}^{+\infty} x d F_{\xi}(x)$, а дисперсией $D \xi=E|\xi-E \xi|^{2}$. Норму случайной величины $\xi$ в $L_{p}$ определяют как $\left(E|\xi|^{p}\right)^{1 / p}$.

Говорят, что случайные величины $\xi_{1}, \xi_{2}, \ldots, \xi_{n}, \ldots$ удовлетворяют ЗБЧ (в форме Чебышева [1], §31), если для $\forall \varepsilon>0$ справедливо соотношение

$$
\lim _{n \rightarrow \infty} P\left\{\left|\frac{1}{n} \sum_{k=1}^{n} \xi_{k}-\frac{1}{n} \sum_{k=1}^{n} E \xi_{k}\right|<\varepsilon\right\}=1,
$$

и удовлетворяет усиленному ЗБЧ (в форме Колмогорова [1], § 34), если

$$
P\left\{\lim _{n \rightarrow \infty}\left(\frac{1}{n} \sum_{k=1}^{n} \xi_{k}-\frac{1}{n} \sum_{k=1}^{n} E \xi_{k}\right)=0\right\}=1 .
$$

В первом случае имеет место сходимость по вероятности, во втором случае сходимость с вероятностью единица (почти наверное). Рассматривают также сходимость в пространствах $L_{p}$.

Как известно, из сходимости почти наверное следует сходимость по вероятности. Также сходимость по вероятности следует из сходимости в $L_{p}$ при $p>0$. Сходимость почти наверное и сходимость в $L_{p}$ не влекут одна другую.

Не оговаривая дополнительно, все неравенства (равенства) для случайных величин в настоящей статье будем понимать как выполненные с вероятностью единица.

Сформулируем в виде леммы теорему Маркова (например, [1], гл. 6, § 34).

Лемма. Пусть для последовательности независимых случайных величин $\left\{\xi_{i}\right\}$ существуют числа $\alpha>1, \beta>0$ такие, что $E\left|\xi_{i}\right|^{\alpha} \leq \beta(i=1,2, \ldots)$. Тогда к последовательности $\left\{\xi_{i}\right\}$ применим закон больших чисел, т.е. $\frac{1}{n} \sum_{i=1}^{n} \xi_{i}-\frac{1}{n} \sum_{i=1}^{n} E \xi_{i} \rightarrow 0$ при $n \rightarrow \infty$ по веролтноcmu.

Теорема 1. Пусть случайные величины $\xi_{1}, \xi_{2}, \ldots, \xi_{n}, \ldots$ попарно независимы, и их абсолютные моменты порядка $p>1$ равномерно ограничены, т.е. существует число $C_{p}>0$ такое, что $E\left|\xi_{i}\right|^{p}<C_{p}(i=1,2, \ldots)$. Тогда имеет место сходимость средних арифметических случайных величин к среднему арифметическому их математических ожиданий в $L_{r}$ nрu $r \in(0, p)$, m.e.

$$
E\left|\frac{1}{n} \sum_{i=1}^{n} \xi_{i}-\frac{1}{n} \sum_{i=1}^{n} E \xi_{i}\right|^{r} \rightarrow 0 \text { npu } n \rightarrow \infty .
$$

Доказательство. Заметим, что в условиях теоремы согласно неравенству моментов ([1], гл. 5, §30) имеем $\left|E \xi_{i}\right| \leq E\left|\xi_{i}\right| \leq\left(C_{p}\right)^{1 / p}$. Так как $\left|\xi_{i}-E \xi_{i}\right|^{p} \leq\left(\left|\xi_{i}\right|+\left|E \xi_{i}\right|\right)^{p} \leq 2^{p-1}\left(\left|\xi_{i}\right|^{p}+\right.$ $\left.\left|E \xi_{i}\right|^{p}\right)$, то для центральных моментов можем записать $E\left|\xi_{i}-E \xi_{i}\right|^{p} \leq 2^{p} C_{p}$.

Ниже используем обозначение $S_{n}=\sum_{i=1}^{n} \xi_{i}$. По лемме (теореме Маркова) случайные величины $X_{n}:=\frac{S_{n}}{n}-E \frac{S_{n}}{n}$ стремятся к нулю при $n \rightarrow \infty$ по вероятности, т. е. $X_{n} \stackrel{P}{\rightarrow} 0$.

Кроме того, по неравенству между средними степенными (например, [3] гл. II, § 2.9) имеем

$$
\left|X_{n}\right| \leq \frac{1}{n} \sum_{i=1}^{n}\left|\xi_{i}-E \xi_{i}\right| \leq\left(\frac{1}{n} \sum_{i=1}^{n}\left|\xi_{i}-E \xi_{i}\right|^{p}\right)^{1 / p} .
$$


Тогда

и по условию

$$
\left|X_{n}\right|^{p} \leq \frac{1}{n} \sum_{i=1}^{n}\left|\xi_{i}-E \xi_{i}\right|^{p}
$$

$$
E\left|X_{n}\right|^{p} \leq \frac{1}{n} \sum_{i=1}^{n} E\left|\xi_{i}-E \xi_{i}\right|^{p} \leq 2^{p} C_{p}
$$

Поэтому высказанное утверждение вытекает из следствия 2 ([4]. гл. III, §9.4).

Этот результат близок к приведенному в ([5], гл. $6, \S 6.2)$, однако в [5] рассмотрен случай одинаковых математических ожиданий $E \xi_{i}=E \xi_{1}(i=1,2, \ldots)$. Теорема 1 будет использована ниже для обоснования ЗБЧ для нелинейных средних.

В условиях теоремы Чебышева ([1], гл. $6, \S 31)$ результат допускает уточнение, а именно, справедливо

Следствие 1. Пусть случайные величины $\xi_{1}, \xi_{2}, \ldots, \xi_{n}, \ldots$ попарно независимы, а их дисперсии равномерно ограничены, т. е. существует число $C>0$ такое, что $D \xi_{i} \leq C^{2}(i=$ $1,2, \ldots)$. Тогда имеет место сходимость средних арифметических случайных величин к среднему их математических ожиданий в $L_{2}$, причем

$$
A_{n}=E\left|\frac{1}{n} \sum_{i=1}^{n} \xi_{i}-\frac{1}{n} \sum_{i=1}^{n} E \xi_{i}\right|^{2} \leq \frac{C^{2}}{n} .
$$

Действительно, в условиях следствия 1

$$
A_{n}=\frac{1}{n^{2}} \sum_{i=1}^{n} D \xi_{i} \leq \frac{C^{2}}{n}
$$

\section{1. ЗБЧ ДЛЯ НЕЛИНЕЙНЫХ СРЕДНИХ ОДИНАКОВО РАСПРЕДЕЛЕННЫХ СЛУЧАЙНЫХ ВЕЛИЧИН}

Пусть случайные величины $\xi_{1}, \xi_{2}, \ldots, \xi_{n}$ одинаково распределены. Рассмотрим, какой вид принимает ЗБЧ для средних общего характера, представленных, например, в ([3], гл. III). Заметим, что средние степенные $\left(\frac{1}{n} \sum_{i=1}^{n} \xi_{i}^{p}\right)^{1 / p}$ при $p>0$ и средняя геометрическая $\sqrt[n]{\prod_{i=1}^{n} \xi_{i}}$ имеют вид

$$
M_{\phi}=\phi^{-1}\left(\frac{1}{n} \sum_{i=1}^{n} \phi\left(\xi_{i}\right)\right),
$$

где $\phi(x)$ есть соответственно $x^{p}$ и $\ln x$, а $\phi^{-1}-$ функция, обратная к $\phi$. Ниже в этом разделе будем рассматривать среднее (1), считая функцию $\phi$ непрерывной и строго монотонной на $(0, \infty)$ (ср. [3], гл. III).

Теорема 2. Пусть функиия $\phi(x)$ непрерывна и строго монотонно возрастает (либо убывает) на $(0, \infty)$. Пусть $\xi_{1}, \xi_{2}, \ldots, \xi_{n}$ - семейство одинаково распределенных, попарно независимых, положстельных случайных величин, и существует конечный момент $E \phi\left(\xi_{1}\right)=b$. Тогда $\phi^{-1}\left(\sum_{i=1}^{n} \frac{1}{n} \phi\left(\xi_{i}\right)\right) \rightarrow \phi^{-1}($ b) при $n \rightarrow \infty$ с вероятноствю 1.

Доказательство. Случайные величины $\phi\left(\xi_{i}\right)$ попарно независимы в силу попарной независимости случайных величин $\xi_{i}$. Кроме того, они одинаково распределены, поскольку таковы случайные величины $\xi_{i}$ и в силу строгой монотонности функции $\phi$. 
Рассмотрим последовательность $Y_{n}=\frac{1}{n} \sum_{i=1}^{n} \phi\left(\xi_{i}\right)$. Так как $\phi\left(\xi_{i}\right)$ одинаково распределены и по условию $E \phi\left(\xi_{i}\right)=b(i=1,2, \ldots)$, то при $n \rightarrow \infty$ по усиленному ЗБЧ в форме Колмогорова (например, [1], §34) $Y_{n} \rightarrow E Y_{n}$ почти наверное. При этом $E Y_{n}=b$. Заметим, что в условиях теоремы на $(0, \infty)$ существует обратная функция $\phi^{-1}$. В силу непрерывности обратной функции заключаем (например, [6], гл. $1, \S 5)$, что $\phi^{-1}\left(Y_{n}\right) \rightarrow \phi^{-1}(b)$ при $n \rightarrow \infty$ почти наверное. Что и требовалось доказать.

Укажем результат, который дает теорема 2 в случае средних степенных, когда $\phi(x)=x^{p}$, a $\phi^{-1}(y)=y^{1 / p}$.

Следствие 2. Пусть $\xi_{1}, \xi_{2}, \ldots, \xi_{n}, \ldots$ - семейство одинаково распределенных, взаимно независимых, положительных случайных величин и существует конечный момент $E\left(\xi_{1}^{p}\right):=$ $b$ при $p>0$. Тогда последовательность средних степенных $Z_{n}=\left(\frac{1}{n} \sum_{i=1}^{n} \xi_{i}^{p}\right)^{1 / p}$ порядка $p>0$ сходится при $n \rightarrow \infty$ к $\sqrt[p]{b}$ почти наверное.

Действительно, в этом случае $\phi\left(\xi_{i}\right)=\xi_{i}^{p}, Y_{n}=\frac{1}{n} \sum_{i=1}^{n} \xi_{i}^{p}$ и $Z_{n}=\phi^{-1}\left(Y_{n}\right)$.

Для средних гармонических, когда $\phi(x)=\ln x$, а $\phi^{-1}(y)=e^{y}$, теорема 2 влечет

Следствие 3. Пусть $\xi_{1}, \xi_{2}, \ldots, \xi_{n}, \ldots$ - семейство одинаково распределенных, взаимно независимых, положительных случайных величин с конечным математическим ожиданием $a>0$. Тогда случайные величины $X_{n}=\sqrt[n]{\prod_{i=1}^{n} \xi_{i}}$, характеризующие средние геометрические, почти наверное сходятся при $n \rightarrow \infty$ к $e^{m}$, где $m:=E \ln \xi_{1}$.

Действительно, в этом случае $\phi\left(\xi_{i}\right)=\ln \xi_{i}, Y_{n}=\frac{1}{n} \sum_{i=1}^{n} \ln \xi_{i}$ и $X_{n}=\phi^{-1}\left(Y_{n}\right)$.

Рассмотрим, какой вид принимает ЗБЧ для средних гармонических. Пусть $\xi_{1}, \xi_{2}, \ldots, \xi_{n}$ - положительные случайные величины. Обозначим через $W_{n}=n\left(\frac{1}{\xi_{1}}+\frac{1}{\xi_{2}}+\cdots+\frac{1}{\xi_{n}}\right)^{-1}=$ $\left(\frac{1}{n} \sum_{i=1}^{n} \frac{1}{\xi_{i}}\right)^{-1}$ среднюю гармоническую случайных величин $\xi_{1}, \xi_{2}, \ldots, \xi_{n}$.

Отметим, что $W_{n}$ представима в виде $\phi\left(\frac{1}{n} \sum_{i=1}^{n} \phi\left(\xi_{i}\right)\right)$ с функцией $\phi(x)=1 / x$ при $x>0$. При этом функция $1 / x$ монотонно убывает на $(0, \infty)$.

Аналогично теореме 2 устанавливается результат для композиций случайных величин вида $g\left(\frac{1}{n} \sum_{i=1}^{n} \phi\left(\xi_{i}\right)\right)$, где $g$ и $\phi-$ заданные числовые функции.

Теорема 3. Пусть функиии $\phi(x)$ и $g(x)$ непрерывны на интервале $(0, \infty)$, причем $\phi$ строго монотонна. Пусть $\xi_{1}, \xi_{2}, \ldots, \xi_{n}, \ldots$ - семейство одинаково распределенных, взаимно независимых, положительных случайных величин, и существует конечный момент $E \phi\left(\xi_{1}\right)=b$. Тогда $g\left(\sum_{i=1}^{n} \frac{1}{n} \phi\left(\xi_{i}\right)\right) \rightarrow g(b)$ при $n \rightarrow \infty$ с вероятностъю 1.

Следствие 4. Пусть $\xi_{1}, \xi_{2}, \ldots, \xi_{n}, \ldots$ - семейство одинаково распределенных, взаимно независимых, положительных случайных величин и существует конечное математическое ожидание $E \frac{1}{\xi_{1}}=\omega$. Тогда последовательность $W_{n}$ сходится почти наверное к $1 / \omega$ при $n \rightarrow \infty$. 
Отметим, что при некоторых дополнительных ограничениях последовательности нелинейных средних сходятся к своим пределам в среднем. Этот вопрос, в общем случае (не одинаково распределенных случайных величин), обсуждается ниже.

\section{2. ЗБЧ ДЛЯ НЕЛИНЕЙНЫХ СРЕДНИХ. ОБЩИЙ СЛУЧАЙ}

Рассмотрим последовательность попарно независимых случайных величин $\xi_{i} \quad i=1,2, \ldots$, не являющихся одинаково распределенными. Пусть скалярная функция $\phi(x)$ задана. Положим $Y_{n}=\frac{1}{n} \sum_{i=1}^{n} \phi\left(\xi_{i}\right)$. При дополнительных ограничениях, например, когда дисперсии случайных величин $\phi\left(\xi_{i}\right)$ равномерно ограничены, по теореме Колмогорова (например, [1], §34) $Y_{n}-\frac{1}{n} \sum_{i=1}^{n} E \phi\left(\xi_{i}\right) \rightarrow 0$ при $n \rightarrow \infty$ почти наверное. Однако отсюда (вообще говоря) не следует сходимость $g\left(Y_{n}\right)-g\left(\frac{1}{n} \sum_{i=1}^{n} E \phi\left(\xi_{i}\right)\right) \rightarrow 0$ при $n \rightarrow \infty$ для заданной непрерывной функции $g$. Так что результат теоремы 3 не допускает прямого переноса на случайные величины, не являющиеся одинаково распределенными. В этом случае справедлива

Теорема 4. Пусть скалярная функиия $\phi(x)$ определена и непрерывна на $(0, \infty)$. Пусть положительные случайные величины $\xi_{1}, \xi_{2}, \ldots, \xi_{n}, \ldots$ попарно независимы, и выполнено условие равномерной ограниченности, т.е. $\phi\left(\xi_{i}\right) \in[\alpha, \beta]$ для некоторого отрезка $[\alpha, \beta]$. Пусть $g(y)$ - непрерывно дифференцируемая на $[\alpha, \beta]$ скалярная функиия. Тогда $g\left(Y_{n}\right)-$ $g\left(E Y_{n}\right) \rightarrow 0$ при $n \rightarrow \infty$ в среднем любого порлдка $r>0$.

Действительно, по условию

$$
g\left(Y_{n}\right)-g\left(E Y_{n}\right)=g^{\prime}\left(Y_{n}+\theta_{n}\left(E Y_{n}-Y_{n}\right)\right)\left(Y_{n}-E Y_{n}\right),
$$

где $\theta_{n} \in(0,1)$.

В силу предположения теоремы $Y_{n} \in[\alpha, \beta]$ и $E Y_{n} \in[\alpha, \beta]$. Пусть $L=\max \left\{g^{\prime}(y)\right\}$ при $y \in[\alpha, \beta]$. Тогда из (2) следует

$$
\left|g\left(Y_{n}\right)-g\left(E Y_{n}\right)\right| \leq L\left|Y_{n}-E Y_{n}\right| .
$$

Заметим, что в наших условиях случайные величины $\phi\left(\xi_{i}\right)$ попарно независимы и $\left|\phi\left(\xi_{i}\right)\right| \leq$ $c:=\max \{|\alpha|,|\beta|\}$. Следовательно, $E\left|\phi\left(\xi_{i}\right)\right|^{p} \leq c^{p}$ для всех $i=1,2, \ldots$, при любом $p>1$. Поэтому теорема 1 влечет сходимость в среднем $Y_{n}-E Y_{n} \rightarrow 0$ при $n \rightarrow \infty$ любого порядка $r>0$.

Возведем обе части формулы (3) в степень $r$ и вычислим математическое ожидание от обеих частей в полученном неравенстве. Тогда с учетом сходимости $Y_{n}-E Y_{n} \rightarrow 0$ в $L_{r}$ при $n \rightarrow \infty$ получим требуемый результат.

Отметим, что в теореме 4, в отличие от теорем 2, 3, не предполагается монотонность функции $\phi$ (поскольку не требуется устанавливать одинаковую распределенность случайных величин $\left.\phi\left(\xi_{i}\right)\right)$.

Теорема 4 является одним из вариантов так называемых теорем непрерывности (например, [6], гл. $1, \S 5)$, представленном в удобном для дальнейшего изложения виде.

Напрямую известные результаты для наших целей не применимы, поскольку предполагают (например, [6]) сходимость в некотором смысле последовательности $E Y_{n}$ (чего, вообще говоря, нет в нашем случае). 
Для последовательности случайных величин $\xi_{1}, \xi_{2}, \ldots$ рассмотрим средние степенные $Z_{n}=\left(\frac{1}{n} \sum_{i=1}^{n} \xi_{i}^{p}\right)^{1 / p}$ при заданном $p>1$. Положим $A_{n}=\left(\frac{1}{n} \sum_{i=1}^{n} E \xi_{i}^{p}\right)^{1 / p}$. Из теоремы 4 вытекает

Теорема 5. Пусть случайные величины $\xi_{1}, \xi_{2}, \ldots, \xi_{n}, \ldots$ положительны, попарно независимы и выполнено соотношение $0<\alpha \leq \xi_{i}^{p} \leq \beta(i=1,2, \ldots)$ для заданного $p>1 u$ некоторых положительных постолнных $\alpha, \beta$. Тогда последовательность случайных величин $Z_{n}=\left(\frac{1}{n} \sum_{i=1}^{n} \xi_{i}^{p}\right)^{1 / p}$ сходится при $n \rightarrow \infty \kappa$ числовой последовательности $A_{n}=$ $\left(\frac{1}{n} \sum_{i=1}^{n} E \xi_{i}^{p}\right)^{1 / p}$ в $L_{r}$ для любого $r>0$.

Действительно, в этом случае $\phi(x)=x^{p}, g(y)=\phi^{-1}(y)=y^{1 / p}$. Положим $Y_{n}=Z_{n}^{p}=$ $\frac{1}{n} \sum_{i=1}^{n} \xi_{i}^{p}$. Тогда $E Y_{n}=A_{n}^{p}=\frac{1}{n} \sum_{i=1}^{n} E \xi_{i}^{p}$, а $Z_{n}=g\left(Y_{n}\right)$ и $A_{n}=g\left(E Y_{n}\right)$.

По условию $0<\alpha \leq \phi\left(\xi_{i}\right) \leq \beta$ для $i=1,2, \ldots$ При этом функция $g^{\prime}(y)=\frac{1}{p} y^{1 / p-1}$ непрерывна на отрезке $[\alpha, \beta]$.

Теперь требуемый результат следует из теоремы 4.

Учитывая конкретный вид средней степенной, можно получить результат о сходимости при меньших ограничениях. А именно, справедлива

Теорема 6. Пусть случайные величины $\xi_{1}, \xi_{2}, \ldots, \xi_{n}, \ldots$ положительны и попарно независимы, а их началъные абсолютные моменты порлдка $q$ при $q>p>1$ равномерно ограничены, т.е. $E\left|\xi_{i}\right|^{q} \leq C(i=1,2, \ldots)$ для некоторой постоянной $C>0$. Тогда последовательность случайных величин $Z_{n}=\left(\frac{1}{n} \sum_{i=1}^{n} \xi_{i}^{p}\right)^{1 / p}$ (для заданного $\left.p>1\right)$ сходится при $n \rightarrow \infty \kappa$ числовой последовательности $A_{n}=\left(\frac{1}{n} \sum_{i=1}^{n} E \xi_{i}^{p}\right)^{1 / p}$ в $L_{s} \partial л я s \in(0, q)$, m. e. $E\left|Z_{n}-A_{n}\right|^{s} \rightarrow 0$ nрu $n \rightarrow \infty$.

Действительно, положим $Y_{n}=Z_{n}^{p}=\frac{1}{n} \sum_{i=1}^{n} \xi_{i}^{p}$ и $a_{n}=A_{n}^{p}=\frac{1}{n} \sum_{i=1}^{n} E \xi_{i}^{p}$. Тогда

$$
\left|Z_{n}-A_{n}\right|=\left|Y_{n}^{1 / p}-a_{n}^{1 / p}\right|
$$

Отсюда по неравенству $\left(a^{1 / p}-b^{1 / p}\right)^{p} \leq a-b$, верному для $\forall a \geq b>0$ и $p>1$, можно записать

$$
\left|Z_{n}-A_{n}\right|^{p}=\left|Y_{n}^{1 / p}-a_{n}^{1 / p}\right|^{p} \leq\left|Y_{n}-a_{n}\right| .
$$

Заметим, что в условиях теоремы $E\left(\left|\xi_{i}\right|^{p}\right)^{q / p}<C(i=1,2, \ldots)$, причем по условию $q / p>1$. Поэтому теорема 1 влечет сходимость $E\left|Y_{n}-a_{n}\right|^{r} \rightarrow 0$ при $r \in(0, q / p)$ и, следовательно, утверждение теоремы 6 .

Рассмотрим ЗБЧ для средних геометрических $\left(\prod_{i=1}^{n} \xi_{i}\right)^{1 / n}$ случайных величин $\xi_{1}, \xi_{2}, \ldots$ Из теоремы 4 вытекает

Теорема 7. Пусть случайные величины $\xi_{1}, \xi_{2}, \ldots, \xi_{n}, \ldots$ положительны и попарно независимы. Пусть для некоторой постоянной с $>0$ выполнено соотночение $\left|\ln \xi_{i}\right| \leq c(\forall i=$ $1,2 \ldots)$. Тогда $\left(\prod_{i=1}^{n} \xi_{i}\right)^{1 / n}-\exp \left\{\frac{1}{n} \sum_{i=1}^{n} E \ln \xi_{i}\right\} \rightarrow 0$ в среднем любого порлдка $r>1$ при $n \rightarrow \infty$. 
Действительно, положим $X_{n}=\left(\prod_{i=1}^{n} \xi_{i}\right)^{1 / n}, Y_{n}=\ln X_{n}=\frac{1}{n} \sum_{i=1}^{n} \ln \xi_{i}, a_{n}=E Y_{n}=\frac{1}{n} \sum_{i=1}^{n} E \ln \xi_{i}$ и $A_{n}=\exp a_{n}$. В этом случае в соответствии с теоремой $4 \phi(x)=\ln x$, а $g(y)=\phi^{-1}(y)=e^{y}$. Поэтому $X_{n}=g\left(Y_{n}\right)$ и $A_{n}=g\left(E Y_{n}\right)$.

По условию $-c \leq \phi\left(\xi_{i}\right) \leq c(i=1,2, \ldots)$, а функция $g(y)$ непрерывно дифференцируема на всей числовой оси. Так что выполнены все условия теоремы 4 и, значит, справедливо заключение теоремы 7 .

Замечание 1. Пусть для некоторых положительных постоянных $\alpha, \beta$ выполнено условие $0<\alpha \leq \xi_{i} \leq \beta(i=1,2, \ldots)$. Тогда найдется постоянная $c>0$, для которой выполнено соотношение $\left|\ln \xi_{i}\right| \leq c(\forall i=1,2, \ldots)$, требуемое в теореме 7 .

Действительно, по условию $\ln \alpha \leq \ln \xi_{i} \leq \ln \beta$ для $i=1,2, \ldots$ Тогда

$$
\left|\ln \xi_{i}\right| \leq \max \{|\ln \alpha|,|\ln \beta|\}:=c .
$$

Учитывая специфику средних геометрических, можно установить справедливость следующего результата.

Теорема 8. Пусть случайные величины $\xi_{1}, \xi_{2}, \ldots, \xi_{n}, \ldots$ положительны и попарно независимы. Пусть для некоторых постолнных $p>1$ и $d>0$ выполнено соотношение $E\left|\ln \xi_{i}\right|^{p} \leq d$ $(\forall i=1,2, \ldots)$. Тогда $\left(\prod_{i=1}^{n} \xi_{i}\right)^{1 / n}-\exp \left\{\frac{1}{n} \sum_{i=1}^{n} E \ln \xi_{i}\right\} \rightarrow 0$ по вероятности при $n \rightarrow \infty$.

Доказательство. Будем использовать те же обозначения для $X_{n}, Y_{n}, a_{n}$ и $A_{n}$, что и в доказательстве теоремы 7.

Заметим, что случайные величины $\ln \xi_{i}$ попарно независимы, поскольку таковы $\xi_{i}$. При этом $E\left|\ln \xi_{i}\right|^{p} \leq d \forall i=1,2, \ldots$ Тогда теорема 1 влечет сходимость $Y_{n}-a_{n} \rightarrow 0$ по вероятности при $n \rightarrow \infty$. То есть $\ln X_{n}-\ln A_{n} \rightarrow 0$ по вероятности. Отсюда $\ln \frac{X_{n}}{A_{n}} \rightarrow 0$ по вероятности. Рассмотрим функцию $\phi(x)=\ln x$ и обратную к ней $\phi^{-1}(y)=\exp \{y\}$. Тогда $\phi^{-1}\left(\ln \frac{X_{n}}{A_{n}}\right)=\frac{X_{n}}{A_{n}} \rightarrow 1$ по вероятности, так как непрерывная функция переводит сходящиеся по вероятности последовательности в такие же. Следовательно, $\frac{1}{A_{n}}\left(X_{n}-A_{n}\right) \rightarrow 0$ по вероятности при $n \rightarrow \infty$.

По предположению и в силу неравенства моментов $\left|E \ln \xi_{i}\right| \leq E\left|\ln \xi_{i}\right| \leq\left(E\left|\ln \xi_{i}\right|^{p}\right)^{1 / p} \leq$ $d^{1 / p}$. Тогда $A_{n} \leq \exp \left\{\frac{1}{n} \sum_{i=1}^{n}\left|E \ln \xi_{i}\right|\right\} \leq \exp \left\{d^{1 / p}\right\}:=\gamma>0$.

Поэтому из предыдущего вытекает сходимость $X_{n}-A_{n} \rightarrow 0$ по вероятности, т. е. утверждение теоремы.

Следствие 5. Пусть выполнены условия теоремы 8 и, дополнительно, математические ожидания случайных величин $\xi_{i}$ равномерно ограничены, т. е. $E \xi_{i} \leq m(\forall i=1,2, \ldots)$ для некоторой постоянной $m>0$. Тогда при $n \rightarrow \infty$ имеет место сходимость $X_{n}-A_{n} \rightarrow 0$ в среднем порядка $r \in(0, p)$.

Действительно, оценим величину $\left|X_{n}\right|^{p}=\left(\prod_{i=1}^{n} \xi_{i}\right)^{p / n}$ при $1<p<n$. В наших предположениях степень $p / n<1$, поэтому она характеризует выпуклую вверх (вогнутую) функцию. Тогда по неравенству Иенсена (например, [7], гл. II, §11.6)

$$
E\left|X_{n}\right|^{p}=E\left(\left(\prod_{i=1}^{n} \xi_{i}\right)^{p / n}\right) \leq\left(E\left(\prod_{i=1}^{n} \xi_{i}\right)\right)^{p / n}=\left(\prod_{i=1}^{n} E \xi_{i}\right)^{p / n} \leq m^{p} .
$$


Здесь воспользовались независимостью случайных величин $\xi_{i}$.

Кроме того, как показано в теореме $8, A_{n} \leq \exp \left\{d^{1 / p}\right\}$. Следовательно, по $c_{r}$-неравенству ([4], гл. III, §9.3) при $r=p>1$ имеем

$$
E\left|X_{n}-A_{n}\right|^{p} \leq 2^{p-1}\left(E\left|X_{n}\right|^{p}+E\left|A_{n}\right|^{p}\right) \leq \text { const. }
$$

Тогда по ([4] гл. III, §9.4, следствие 2) получим высказанное утверждение.

Замечание 2. В условиях следствия 5 имеет место сходимость при $n \rightarrow \infty$ средних геометрических в $L_{r}$ произвольного конечного порядка $r \in(0, \infty)$.

Действительно, пусть $r>1$. Конечное число начальных членов не влияет на сходимость. Поэтому можем рассматривать члены последовательности $X_{n}$, начиная с $n>r$. Тогда аналогично следствию 5

$$
\sup _{n>r} E\left|X_{n}\right|^{r}<\infty .
$$

Это обеспечивает справедливость замечания 2.

Рассмотрим случай средних гармонических $W_{n}=n\left(\frac{1}{\xi_{1}}+\frac{1}{\xi_{2}}+\cdots+\frac{1}{\xi_{n}}\right)^{-1}=\left(\frac{1}{n} \sum_{i=1}^{n} \frac{1}{\xi_{i}}\right)^{-1}$ случайных величин $\xi_{1}, \xi_{2}, \ldots$ Положим

$$
B_{n}=n / \sum_{i=1}^{n} E \frac{1}{\xi_{i}} .
$$

Теорема 4 влечет следующее утверждение.

Теорема 9. Пусть случайные величины $\xi_{1}, \xi_{2}, \ldots, \xi_{n}, \ldots$ попарно независимы и удовлетворяют соотношениям $0<\alpha \leq \xi_{i} \leq \beta(i=1,2, \ldots)$ для некоторых постолнных $\alpha$, $\beta$. Тогда $W_{n}-B_{n} \rightarrow 0$ при $n \rightarrow \infty$ в среднем в $L_{r}$ при всяком $r>0$.

Действительно, в этом случае $\phi(x)=x^{-1}, g(y)=y^{-1}$. Положим $Y_{n}=\frac{1}{n} \sum_{i=1}^{n} \frac{1}{\xi_{i}}$. Тогда $E Y_{n}=\frac{1}{n} \sum_{i=1}^{n} E \frac{1}{\xi_{i}}$ и $g\left(Y_{n}\right)=W_{n}$, а $g\left(E Y_{n}\right)=B_{n}$. По условию $1 / \beta \leq \phi\left(\xi_{i}\right) \leq 1 / \alpha$. Поскольку производная $g^{\prime}(y)=\left(y^{-1}\right)^{\prime}=-1 / y^{2}$ непрерывна для $y \in[1 / \beta, 1 / \alpha]$, то высказанный результат следует из теоремы 4.

Замечание 3. На самом деле результат теоремы 9 справедлив в предположении, что найдутся такие постоянные $A, B, \varepsilon>0$, что $\xi_{i} \in[-A,-\varepsilon] \cup[\varepsilon, B](i=1,2, \ldots)$.

В заключение приведем краткий сравнительный анализ результатов настоящей работы в рамках современного состояния развития "нелинейного ЗБЧ".

Одно из направлений развития "нелинейного ЗБЧ” связано с мартингалами. Для них установлен обобщенный ЗБЧ (например, [8], гл. VII, $\S 9,10)$. Для нелинейных средних, рассмотренных выше, эта методика не применима.

Схема прямого обобщения классического ЗБЧ на нелинейный случай представлена в [9]. При этом рассматривается класс нелинейностей $f_{n}$ с ограниченным градиентом $\left\|\nabla f_{n}(x)\right\| \leq \gamma_{n}\left(x \in R^{n}:|x| \leq 1\right)$. В предположении, что $\gamma_{n} \rightarrow 0$ при $n \rightarrow \infty$ обосновывается ЗБЧ. Случай нелинейных средних случайных величин результатом из [9] не охватывается.

Подчеркнем, что наш подход позволяет указать постоянные $a_{n}$, к которым стабилизируются $f_{n}$, соответствующие нелинейным средним указанного вида. А именно, в нашем случае 
$f_{n}$ представимы в виде $f_{n}=g\left(\frac{1}{n} \sum_{i=1}^{n} \phi\left(\xi_{i}\right)\right)$, где $g$ и $\phi-$ числовые функции, порождаемые соответствующими нелинейными средними. Показано, что $a_{n}=g\left(\frac{1}{n} \sum_{i=1}^{n} E \phi\left(\xi_{i}\right)\right)$.

В математической статистике активно изучают асимптотические методы для различных классов нелинейных задач. Отметим, в частности, метод локальной линеаризации (например, [10]). Однако этот метод в нашей ситуации не применим, так как в противном случае в качестве постоянных $a_{n}$ выступали бы $a_{n}=f\left(E \xi_{1}, E \xi_{2}, \ldots, E \xi_{n}\right)$, что не согласуется с нашими результатами.

\section{ЛитературА}

[1] Гнеденко Б.В. Курс теории вероятностей (Наука М., 2005).

[2] Джини К. Средние величинъ (Статистика, М., 1970).

[3] Харди Г.Г., Литтлвуд Дж. Е., Полиа Г. Неравенства (Ин. лит., М., 1948).

[4] Лоэв М. Теория вероятностей (Ин. лит., М., 1962).

[5] Уиттл П. Вероятность (Наука, М., 1982).

[6] Боровков А.А. Математическая статистика (Наука, М., 1984).

[7] Невё Ж. Математические основы теории вероятностей (Мир, М., 1969).

[8] Феллер В. Введение в теорию вероятностей и ее приложенил. Т. 2 (Мир, М., 1984).

[9] Опойцев В.И. Нелинейнъй закон болъших чисел, Автоматика и телемеханика, № 4, 65-75 (1994).

[10] Барндорф-Нильсен О., Кокс Д. Асимптотические методы в математической статистике (Мир, М., 1999).

Владимир Львович Хацкевич

Военный учебно-научный центр Военно-воздушных сил

"Военно-воздушная академия им. Н.Е. Жуковского и Ю.А. Гагарина",

ул. Старых Большевиков, д. 54 а, г. Воронеж, 394064, Россия,

e-mail : vlkhats@mail.ru

\section{V.L. Khatskevich}

\section{On the law of large numbers for nonlinear mean random variables}

Abstract. We consider variants of the law of large numbers with respect to the average power, average geometric and mean harmonic systems of random variables and estabilihs various forms of convergence of the indicated means to the limiting values.

Keywords: random variables, nonlinear averages, the law of large numbers.

Vladimir L'vovich Khatskevich

Military educational scientific center Air force

"Air force academy named after N.E. Zhukovsky and Y.U. Gagarin",

54 a old Bolsheviks str., Voronezh, 394064 Russia,

e-mail:vlkhats@mail.ru 